\title{
Inner hair cell responses to the velocity of basilar membrane motion in the guinea pig
}

\author{
ALFRED L. NUTTALL, M. CHRISTIAN BROWN, ROBERT I. MASTA and \\ MERLE LAWRENCE
}

Kresge Hearing Research Institute, University of Michigan Medical School, 1301 E. Ann Street, Ann Arbor, Mich. 48109 (U.S.A.)

(Accepted December 4th, 1980)

Key words: intracellular recording - cochlea - inner hair cell - basilar membrane - organ of Corti - velocity response - displacement response - guinea pig

Triangular wave acoustic stimulation at $200 \mathrm{~Hz}$ produced the expected square wave cochlear microphonic at the round window membrane and within the scala media. Intracellular recordings from inner hair cells (IHC) of the first cochlear turn showed a combination waveform having both spike impulse and square wave features. The IHC response suggests a sensitivity of these cells to both the displacement and to the velocity of basilar membrane motion.

Inner hair cells (IHC) which respond to the velocity of basilar membrane motion have recently been reported by Sellick and Russell ${ }^{5}$. Velocity response of IHCs was suggested by Dallos et al. ${ }^{2}$, based on study of the cochlear microphonic in Kanamycin treated guinea pigs, and it was subsequently observed in the pattern of activity of afferent neurons of normal gerbils? ${ }^{7}$. The present report offers some further observations on the nature of the IHC velocity response in normal guinea pigs.

Albino guinea pigs with body weights between 200 and $300 \mathrm{~g}$ were anesthetized with Innovar* $(0.8 \mathrm{ml} / \mathrm{kg}$ i.m. $)$ or a pentobarbital $(15 \mathrm{mg} / \mathrm{kg}$ i.p. $) /$ Innovar $(0.4 \mathrm{ml} / \mathrm{kg}$ i.m.) combination. Atropine and curare were usually given as well. Supplemental heating was supplied to maintain the rectal temperature at approximately $38.5^{\circ} \mathrm{C}$. Heart rate and end-respiratory $\mathrm{CO}_{2}$ were also monitored. A ventral-postauricular surgical dissection was carried out to expose the cochlea, and a small fenestra was made into the scalia tympani of the basal turn according to the method of Sellick and Russell ${ }^{4}$. Glass micropipette electrodes for intracellular measurements had resistances of approximately $200 \mathrm{M} \Omega$ when filled with a solution of $4 \%$ Procion blue dye in $150 \mathrm{mM}$ $\mathrm{KCl}$. For some experiments, electrodes were beveled using the thick slurry beveling technique $^{3}$. A hydraulic microdrive and a piezoelectric driver were used to advance the electrodes. Electrode signals were amplified by a capacity-compensated DC preamplifier and photographed from a storage oscilloscope. A cell was judged to be an inner

* Innovar - Fentanyl and Droperidol, Pittman-Moore. 


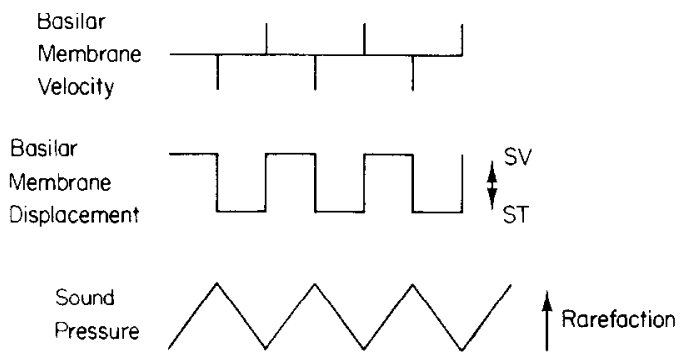

Fig. 1. The idealized (displacement) motion of the basilar membrane to low-frequency acoustic triangular wave stimulation compared to the velocity of the motion.

I

NON-SENSORY

CELL
II

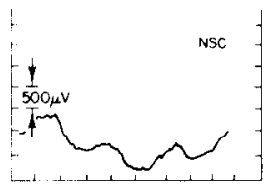

ORGAN OF

CORTI
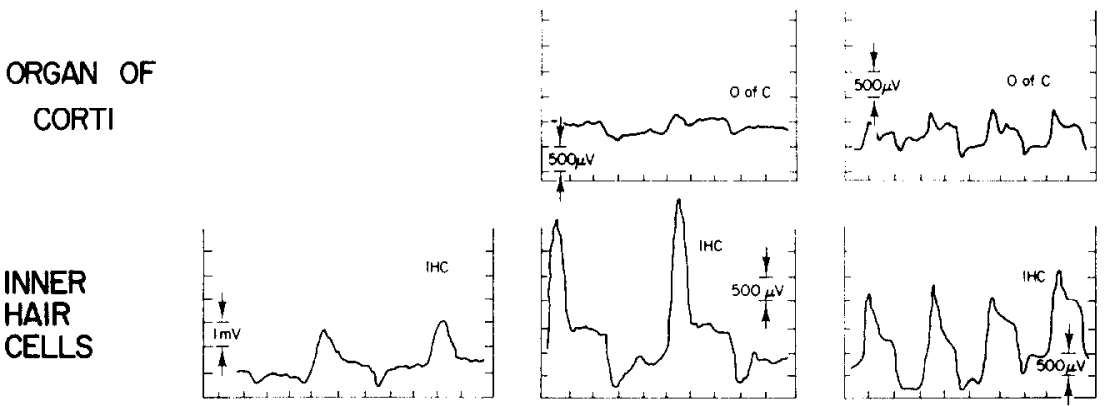

SCALA

MEDIA
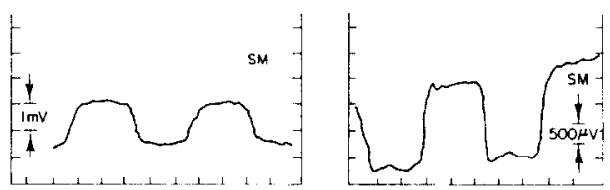

SOUND PRESSURE
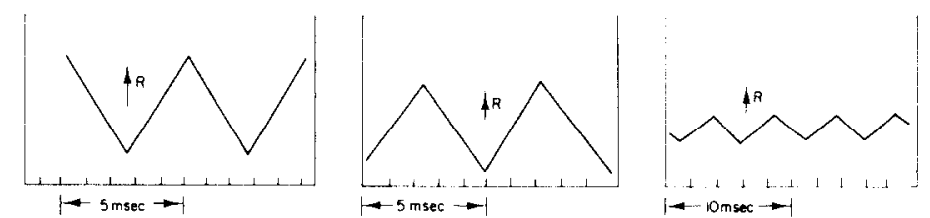

Fig. 2. Intracochlear recordings of responses to low-frequency acoustic triangular wave stimulation are compared for 3 guinea pigs (I and II, $105 \mathrm{~dB}$ SPL; III, $95 \mathrm{~dB}$ SPL). NSC, the intracellular response of a non-sensory cell; $O$ of $C$, the extracellular response within the organ of Corti; IHC, the intracellular response of an inner hair cell; SM, the response within the endolymph of scala media; $\mathbf{R} \uparrow$, the direction of acoustic rarefaction. 
hair cell at the time of recording by: (1) the depth of penetration and the visually observed placement of the electrode into the organ of Corti; (2) the magnitude of the resting membrane potential (approximately $-30 \mathrm{mV}$ ); and (3) the increase in magnitude of the summating potential, evoked by a tone burst near the characteristic frequency (14-19 kHz), as the electrode penetrated the cell. Additionally, some cells were marked by intracellular dye injection for later histological identification. (However, the cells of Fig. 2 were not dye marked.) The experiments were carried out on a vibration isolation table in a sound-and electrically-shielded booth.

Acoustic stimuli were produced by a $0,5 \mathrm{in}$. Bruel and Kjaer condenser microphone, driven by a distortion compensating amplifier system. Sound was coupled into the external ear canal with an acrylic plastic speculum. A $200 \mathrm{~Hz}$ triangular sound pressure wave was used as a stimulus designed to separate velocity from displacement responses. For this acoustic stimulus, the idealized basilar membrane displacement follows the stapes volume velocity ${ }^{1}$, which is the first derivative with respect to time of the sound pressure and has a square wave shape (Fig. 1). The basilar membrane velocity, which is the second derivative with respect to time, would then appear as bipolar impulses or spikes.

Fig. 2 shows potentials recorded from 3 cells, in 3 guinea pigs, as a microelectrode is advanced through the organ of Corti, into an inner hair cell, and finally into scala media. Potentials recorded close to the basilar membrane have the square wave shape of the expected basilar membrane displacement. As the microelectrode approaches and penetrates an inner hair cell, the recorded waveform becomes much more impulse-like, which is characteristic of a response to basilar membrane velocity. The response is asymmetrical, being 3-5 times larger during the positive phase (depolarization) than during the negative. There is a small increase in the square wave response as well, indicating some response to basilar membrane displacement in the inner hair cell. These responses are typical of 9 inner hair cells which have been recorded. As the electrode enters scala media, the square wave response dominates. Also note the expected phase reversal of the gross cochlear microphonic (CM) potential ${ }^{6}$.

Sellick and Russel1 ${ }^{5}$ also observed velocity response from inner hair cells and additionally reported a frequency dependence of response. Using sinusoidal stimuli, they found IHC response to the velocity of the basilar membrane for frequencies below about $200 \mathrm{~Hz}$, changing to displacement response at higher frequencies. The 200 $\mathrm{Hz}$ triangular wave used in the present study produced a response showing characteristics of both velocity and displacement sensitivity. Here, the velocity response is produced by the $200 \mathrm{~Hz}$ fundamental frequency, while the higher harmonics contributed to the displacement response. However, note that within a non-sensory organ of Corti cell there is a certain amount of square wave cochlear microphonic. This suggests that within the IHC, a portion of the receptor potential is not generated within the cell itself, but rather is passively conducted across the cell membrane.

The progression of signals shown in Fig. 2, cell II, demonstrates how specific and localized the IHC response can be. The signal obtained extracellularly within the organ of Corti, and the signal from within the scala media exhibit very little velocity 
character compared to the intracellular response of the IHC, although all 3 signals were obtained successively along a single electrode penetration path. Nevertheless, we have observed that localized IHC activity can be found in extracellular recordings when the electrode is close to an inner hair cell (Fig. 2, cell III).

As a final note, we point out that while our data and that of Sellick and Russell ${ }^{5}$ show IHC depolarization only for velocity in one direction (toward scala vestibuli), recordings from high frequency ( $\mathrm{CF}>5 \mathrm{kHz}$ ) auditory neurons by Zwislocki and Sokolich 8 show excitatory responses to velocity in both directions. Thus, the IHC velocity response findings seem to support the Zwislocki and Sokolich ${ }^{8}$ conclusion that outer hair cells contribute significantly to afferent nerve velocity responses.

This work was supported by Public Health Service Grants NS-05785, NS-07106 and NS-15107.

1 Dallos, P., The Auditory Periphery, Academic Press, New York, 1973.

2 Dallos, P., Billone, M. C., Durrant, J. D. and Raynor, C.-Y. W. S., Cochlear inner and outer hair cells: functional differences, Science, 177 (1972) 356-358.

3 Lederer, W. J., Spindler, A. J. and Eisner, D. A., Thick slurry beveling: a new technique for beveling extremely fine microelectrodes and micropipettes, Pflüger's Arch., 381 (1979) 287-288.

4 Sellick, P. M. and Russell, I. J., Intracellular studies of the receptor potential of the inner hair cells of the guinea pig cochlea: techniques. In H. A. Beagley (Ed.), Auditory Investigation: The Scientific and Technological Basis, Clarendon Press, Oxford, 1979.

5 Sellick, P. M. and Russell, I. J., The responses of inner hair cells to basilar membrane velocity during low frequency auditory stimulation in the guinea pig cochlea, Hearing Res., 2 (1980) 439-445.

6 Tasaki, I., Davis, H. and Eldredge, D. H., Exploration of cochlear potentials in guinea pig with a microelectrode, J. acoust. Soc. Amer., 26 (1954) 765-773.

7 Zwislocki, J. J. and Sokolich, W. G., Velocity and displacement responses in auditory-nerve fibers, Science, 182 (1973) 64-66.

8 Zwislocki, J. J. and Sokolich, W. G., Neuro-mechanical frequency analysis in the cochlea. In E. Zwicker and E. Terhardt (Eds.), Facts and Models in Hearing, Springer-Verlag, New York, 1974, pp. $107-117$. 